\title{
Recurrent Glioma and Crossed Cerebellar Diaschisis in a Patient Examined With ${ }^{18} \mathrm{~F}-\mathrm{DOPA}$ and ${ }^{18} \mathrm{~F}-\mathrm{FDG} \mathrm{PET} / \mathrm{CT}$
}

\author{
Ferdinando Calabria, MD, $*$ and Orazio Schillaci, $M D^{* \dagger}$
}

\begin{abstract}
Two years after resection of a left parietal glioma, a 46-year-old woman underwent ${ }^{18} \mathrm{~F}$-FDG and ${ }^{18} \mathrm{~F}$-DOPA brain PET/CT. FDG showed left parietal hypometabolism with crossed cerebellar diaschisis. No abnormally increased FDG activity was seen. ${ }^{18} \mathrm{~F}$-DOPA PET/CT scan demonstrated focal left parietal uptake. Recurrent glioma was confirmed by surgical biopsy. ${ }^{18} \mathrm{~F}$-DOPA may demonstrate abnormal amino acid metabolism in evaluation of recurrence of glioma.
\end{abstract}

Key Words: ${ }^{18} \mathrm{~F}-\mathrm{DOPA},{ }^{18} \mathrm{~F}-\mathrm{FDG}, \mathrm{PET} / \mathrm{CT}$, brain tumor recurrence, crossed cerebellar diaschisis

(Clin Nucl Med 2012;37: 878-879)

Received for publication February 16, 2012; revision accepted May 30, 2012.

From the *Department of Nuclear Medicine and Molecular Imaging, IRCCS Neuromed, Pozzilli (IS); and †Department of Biopathology and Diagnostic Imaging, University "Tor Vergata," Rome, Italy.

Conflicts of interest and sources of funding: none declared.

Reprints: Ferdinando Calabria, MD, Department of Nuclear Medicine and Molecular Imaging, IRCCS Neuromed, Via Atinense 18, 86077 Pozzilli (IS), Italy.

E-mail: ferdinandocalabria@hotmail.it.

Copyright (C) 2012 by Lippincott Williams \& Wilkins

ISSN: 0363-9762/12/3709-0878

\section{REFERENCES}

1. Tripathi M, Sharma R, Varshney R, et al. Comparison of F-18 FDG and C-11 methionine for the evaluation of recurrent primary brain tumors. Clin Nucl Med. 2012;37:158-163.

2. Kanhaiya LA, Bhagwant RM, Anish B, et al. Crossed cerebellar diaschisis on F-18 FDG PET/CT. Indian J Nucl Med. 2011;26:102-103.

3. Shih WJ, Huang WS, Milan PP. F-18 FDG PET demonstrates crossed cerebellar diaschisis 20 years after stroke. Clin Nucl Med. 2006;31:259-261.

4. Gonzalez-Forero M, Prieto E, Domingues I, et al. Dual time point ${ }^{18} \mathrm{~F}$-DOPA PET as a tool for characterizing brain tumors. Rev Esp Med Nucl. 2011; 30:88-93.

5. Fueger BJ, Czernin J, Cloughesy $\mathrm{T}$, et al. Correlation of $6-{ }^{18} \mathrm{~F}$-fluoro-L-DOPA PET uptake with proliferation and tumor grade in newly diagnosed and recurrent gliomas. Clin Nucl Med. 2011;36:650-655.

6. Tripathi M, Sharma R, D'Souza M, et al Comparative evaluation of F-18 FDOPA, F-18 FDG and F-18 FLT-PET/CT for metabolic imaging of low grade gliomas. Clin Nucl Med. 2009;34:878-883.

7. Santra A, Kumar R, Sharma P, et al. Detection of recurrence in glioma: a comparative study between Tc-99m GHA SPECT and F-18 FDG PET/CT. Clin Nucl Med. 2011;36:650-655.

8. Chen W, Silverman DH, Delaloye $\mathrm{S}$, et al. ${ }^{18}$ F-FDOPA PET imaging of brain tumors: comparison study with ${ }^{18}$ F-FDG PET and evaluation of diagnostic accuracy. J Nucl Med. 2006;47:904-911.

9. Ledezma CJ, Chen W, Sai V, et al. ${ }^{18}$ F-DOPA PET/MRI fusion in patients with primary/recurrent gliomas: initial experience. Eur J Radiol. 2009;71:242-248.

10. Calabria F, Chiaravalloti A, Di Pietro B, et al. Molecular imaging of brain tumors with 18F-DOPA PET and PET/CT. Nucl Med Commun. 2012; $33: 563-570$. 


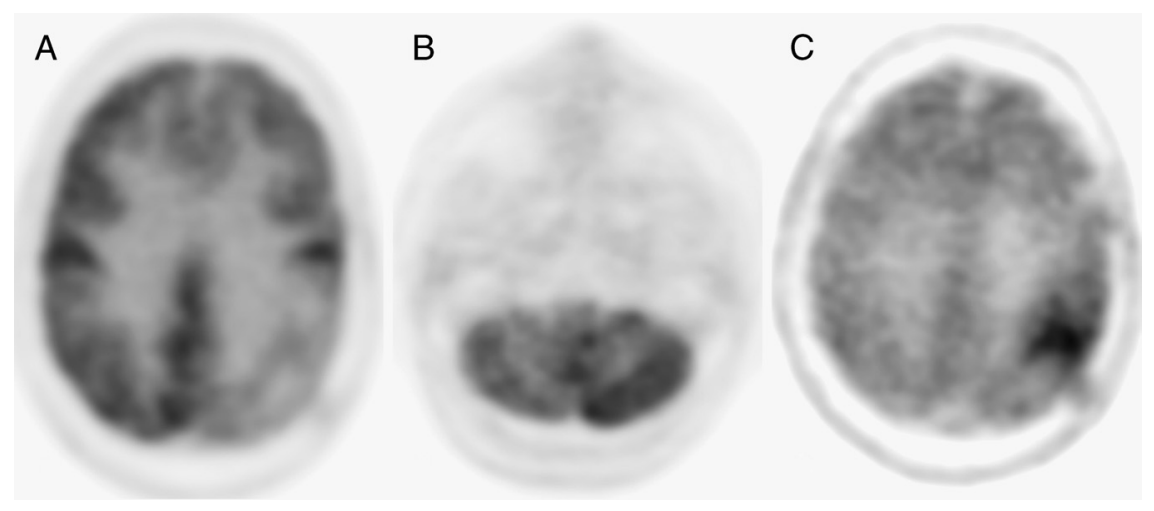

FIGURE 1. In a patient submitted 2 years before to surgical intervention in the left posterior parietal lobe and subsequent radiotherapy for a malignant glioma, axial ${ }^{18}$ F-FDG PET view shows area of hypometabolism in the surgical site (A). No foci of increased uptake of the tracer were detected in the rest of the brain, whereas corresponding CT images, obtained for attenuation correction, ${ }^{1}$ did not show significant morphological abnormalities, with the exception of diffuse hypodensity in the same area, considered as a postsurgical lacuna. A further area of hypometabolism was detected in the right cerebellar hemisphere as shown in axial ${ }^{18}$ F-FDG PET view (B). Considering that the limitation of the excitatory output from an injured brain region could reduce the physiological activity in connecting the cerebellar region contralateral to the lesion site, ${ }^{2,3}$ we interpreted this findings as indicative of crossed cerebellar diaschisis. ${ }^{18} \mathrm{~F}-\mathrm{DOPA}$ PET/CT of the brain, subsequently performed, exhibited in the left posterior parietal lobe an area of focal increased uptake, with SUVmax $=5,{ }^{4}$ suggestive for recurrence of glioma, ${ }^{5}$ as evident in the axial detail (C). No other abnormalities were detected with this radiopharmaceutical.Histological examination confirmed our suspicion and diagnosed tumor recurrence. ${ }^{18} \mathrm{~F}$-FDG PET/CT was not able to identify the tumor recurrence. In fact, some previous articles on this topic well described the limits of this tracer in the evaluation of tumor recurrence, compared with the feasibility of ${ }^{18} \mathrm{~F}-\mathrm{DOPA}$ and ${ }^{18} \mathrm{~F}$-FLT, ${ }^{6}$ or the effectiveness of SPECT with ${ }^{99 \mathrm{~m}} \mathrm{Tc}$-glucoheptonate. ${ }^{7}$ Therefore, ${ }^{18} \mathrm{~F}$-FDG may play a limited role in this field for the intrinsic property of metabolic characterization, allowing the detection of functional disorders such as crossed cerebellar diaschisis. On the other hand, this report confirms the feasibility of ${ }^{18} \mathrm{~F}$-DOPA acting as a tracer of amino acid metabolism in the evaluation of recurrence of brain tumors after surgery and/or radiotherapy, as well known in various articles, because of the possibility to distinguish recurrent neoplasm from radiation necrosis, especially in comparison with ${ }^{18} \mathrm{~F}_{\text {-FDG }} \mathrm{PET}^{8}{ }^{8}$ and by virtue of the low physiological uptake in normal white and gray matter, considering the exception of the basal ganglia.9,10 